\title{
On Inverse Eigenvalue Problems of Quadratic Palindromic Systems with Partially Prescribed Eigenstructure
}

\author{
Kang Zhao*, Lizhi Cheng, Anping Liao and Shengguo Li
}

\begin{abstract}
The palindromic inverse eigenvalue problem (PIEP) of constructing matrices $A$ and $Q$ of size $n \times n$ for the quadratic palindromic polynomial $P(\lambda)=$ $\lambda^{2} A^{\star}+\lambda Q+A$ so that $P(\lambda)$ has $p$ prescribed eigenpairs is considered. This paper provides two different methods to solve PIEP, and it is shown via construction that PIEP is always solvable for any $p(1 \leq p \leq(3 n+1) / 2)$ prescribed eigenpairs. The eigenstructure of the resulting $P(\lambda)$ is completely analyzed.
\end{abstract}

\section{Introduction}

In this paper, we consider the following quadratic palindromic eigenvalue problem:

$$
P(\lambda) x \equiv\left(\lambda^{2} A^{\star}+\lambda Q+A\right) x=0,
$$

where $A$ and $Q$ are $n \times n$ (real or complex) matrices and $Q^{\star}=Q$. The scalar $\lambda$ and nonzero vector $x$ satisfying (1.1) are called an eigenvalue of $Q(\lambda)$ and the (right) eigenvector corresponding to $\lambda$, respectively. Together, $(\lambda, x)$ is called an eigenpair of $Q(\lambda)$. To be specific, we summarize the names and structures of the palindromic system $P(\lambda)$ in 1.1 as follows:

$$
\begin{array}{lll}
\text { T-palindromic } & P(\lambda)=\lambda^{2} A^{\top}+\lambda Q+A, & Q^{\top}=Q, \\
\text { *-palindromic } & P(\lambda)=\lambda^{2} A^{*}+\lambda Q+A, & Q^{*}=Q .
\end{array}
$$

The palindromic quadratic eigenvalue problem (PQEP) 16, 17, 21] is to find $\lambda$ and $x$ such that 1.1 holds. The PQEPs arise in the analysis and numerical solution of high order systems of ordinary and partial differential equations, and enjoy a variety of

Received March 8, 2018; Accepted January 21, 2019.

Communicated by Ren-Cang Li.

2010 Mathematics Subject Classification. 65F18, 65F15, 15A24, 15A29.

Key words and phrases. quadratic palindromic system, inverse eigenvalue problem, partially prescribed eigendata.

Supported by NSFC under Grant Nos. 11371073, 11401580, Research Foundation of Education Department of Hunan Province, China (Grant No. 16C0037), Natural Science Foundation of Hunan Province, China (Grant No. 806175290082) and Research Foundation of National University of Defense Technology, China (Grant No. ZK18-03-01).

*Corresponding author. 
applications. For example, the T-palindromic PQEP was first raised in the study of the vibration analysis of high speed trains in Germany [16, 17], associated with the company $\mathrm{SFE} \mathrm{GmbH} \mathrm{in} \mathrm{Berlin.} \mathrm{Existing} \mathrm{fast} \mathrm{train} \mathrm{systems,} \mathrm{like} \mathrm{the} \mathrm{Chinese} \mathrm{CHR,} \mathrm{the} \mathrm{Japanese}$ Shinkansen, the French TGV and the German TCE, are being modernized and expanded. Vibration is produced from the interaction between the wheels of a train and the rails underneath. Due to the ever increasing speed (currently up to $300 \mathrm{~km} / \mathrm{hr}$ ) of modern trains, the study of its vibration becomes an important task. Research does not only contribute towards the increased comfort of passengers, in terms of lower noise and vibration levels. More importantly, the safety in the operation of the trains will be improved, and the operational and construction costs will be optimized [17, 18,24, 25]. Quadratic real and complex $T$-palindromic PQEPs also arise in the mathematical modeling and numerical simulation of the behaviour of periodic surface acoustic wave (SAW) filters [29]. The computation of the Crawford number [15], associated with the perturbation analysis of symmetric generalized eigenvalue problems, produces an *-palindromic PQEP. For more examples, we refer readers to [8,25] and references therein.

The structure of the coefficient matrices of (1.1) results in a structure in the spectrum. Indeed, transposing the palindromic problem (1.1) yields $\left(\lambda^{\star}\right)^{2} x^{\star} P\left(1 / \lambda^{\star}\right)=0$, which implies that if $\lambda$ is an eigenvalue and $x$ an associated right eigenvector, then $1 / \lambda^{\star}$ is also an eigenvalue with $x^{\star}$ as its left eigenvector. Then it follows that the eigenvalues of the quadratic palindromic system occur in pairs $\left(\lambda, 1 / \lambda^{\star}\right)$. This property is sometimes called "symplectic spectral symmetry". Moreover, the algebraic (geometric and partial) multiplicities of eigenvalues in each pair are equal [25].

In mathematical modelling, we generally assume that there is a correspondence between the endogenous variables and the exogenous variables, for example, the internal parameters and the external behavior. The direct problem is to analyze and derive the spectral information and the dynamical behavior of a system from a priori known physical parameters such as mass, elasticity, length, and so on. Conversely, the inverse problem is to validate, determine or estimate the parameters of the system according to its observed or expected behavior. The inverse problem is just as important as the direct problem in applications. This paper concerns the inverse problem of PQEP.

PQEP is usually solved via two steps: first, transform the PQEP into a general eigenvalue problem (GEP) via linearization [24,25]; second, apply certain numerical methods for GEP. However, GEP may not reflect the symplectic property of spectrum, so their use for numerical computation in such situations may be problematic. Recently, great efforts have been made to the development of structure-preserving numerical method that preserve the symplectic spectrum symmetry, for example, the implicit QR method [19], the hybrid method computing the anti-triangular Schur form 26, the URV decomposition based 
structured method [27, 28], and the structure preserving doubling algorithms [14,22, 23].

The inverse eigenvalue problem (IEP) is to determine the coefficient matrices with partial or entire eigenvalues and eigenvectors prescribed. Many efforts have been devoted to the IEP of quadratic symmetric systems, for example, $2,3,6,9,11,12,20,30,32$ and references therein. The IEP of a palindromic system with entire eigenvalues prescribed is considered in [1]. The IEP for a palindromic matrix polynomial eigenvalue problem is considered without \pm 1 as prescribed eigenvalues [4]. Recently, by using the spectral decomposition, Cai [5] considers the IEP for a quadratic $\star$-(anti)-palindromic system with entire/partial eigenpairs. Zhao [31] considered the model updating problems of the $\star-$ palindromic quadratic system. Their results concentrate on the case that the leading coefficient matrix of the palindromic polynomial is nonsingular.

For simplicity, we make the following assumption throughout this paper.

A1 The prescribed eigenvector matrix $X \in \mathbb{C}^{n \times p}$ is of full rank.

In this paper, under Assumption A1, we consider the IEP of palindromic system (1.1) with $p$ prescribed eigenpairs which can be stated as the following problem.

Problem PIEP. Given eigen-matrix pairs $(\Lambda, X) \in \mathbb{C}^{p \times p} \times \mathbb{C}^{n \times p}(1 \leq p \leq 2 n)$, find matrices $Q, A \in \mathbb{C}^{n \times n}$ such that $Q^{\star}=Q$ and the equation

$$
A^{\star} X \Lambda^{2}+Q X \Lambda+A X=0
$$

is satisfied.

The main contributions of this paper are: two different methods are proposed to solve Problem PIEP; with the partial prescribed eigenpairs, it is proved that the problem is always solvable and the general regular solutions of the problem are characterized via construction; the leading coefficient matrix of regular solutions is not necessarily nonsingular; the eigenstructure of the resulting pencils are completely analyzed.

This paper is organized as follows. In Section 2, we first give a general solution of Problem PIEP with any $p$ prescribed eigenpairs. For $p \leq n$, we proposed several sufficient conditions on the existence of regular solutions to the problem. In Section 3, we solve the Problem PIEP by the QR decomposition of $X$. First, the necessary conditions for the solvability of problem is considered. Then it is shown that the Problem PIEP $(1 \leq$ $p \leq(3 n+1) / 2)$ is always solvable under Assumption A1. Some numerical examples are presented in Section 4 to illustrate the performance of the methods proposed in Sections 2 and 3. Some concluding remarks are drawn in Section 5. 


\section{Solutions of Problem PIEP for $p \leq n$}

Since $p \leq n$, it follows from Assumption A1 and $X \in \mathbb{C}^{n \times p}$ that $X$ is of full column rank, i.e., $\operatorname{rank}(X)=p$. Now, we consider the null space $\mathcal{N}(\Omega)$ of the matrix

$$
\Omega:=\left(\begin{array}{ll}
X^{\star} & \Lambda^{\star} X^{\star}
\end{array}\right) \in \mathbb{C}^{p \times 2 n} .
$$

Denote the dimension of $\mathcal{N}(\Omega)$ by $m$. Since $X$ has linearly independently columns, $m=$ $2 n-p$. In [10], a quadratic symmetric polynomial was characterized by the basis of $\mathcal{N}(\Omega)$. In this section, we adopt their method to characterize a quadratic palindromic polynomial with $(\Lambda, X)$ as its eigendata. Let the columns of

$$
\left(\begin{array}{c}
U^{\star} \\
V^{\star}
\end{array}\right) \in \mathbb{C}^{2 n \times m}
$$

form a basis of the subspace $\mathcal{N}(\Omega)$, where $U, V \in \mathbb{C}^{m \times n}$. Then the equation

$$
\left(X^{\star}, \Lambda^{\star} X^{\star}\right)\left(\begin{array}{c}
U^{\star} \\
V^{\star}
\end{array}\right)=0
$$

holds. Define the quadratic palindromic polynomial $P(\lambda)$ be the one with

$$
A=V^{\star} U, \quad Q=U^{\star} U+V^{\star} V
$$

We claim that the above definitions are sufficient for constructing a solution to Problem PIEP.

Theorem 2.1. Given any matrix pair $(\Lambda, X) \in \mathbb{C}^{p \times p} \times \mathbb{C}^{n \times p}(p \leq n)$, let $(U, V)$ be any solution to (2.2) such that the columns of 2.1 form a basis of $\mathcal{N}(\Omega)$. Then $(\Lambda, X)$ is an eigenpair of the quadratic palindromic polynomial

$$
P(\lambda)=\lambda^{2} A^{\star}+\lambda Q+A,
$$

with coefficients $A$ and $Q$ defined by (2.3).

Proof. Since $(U, V)$ satisfies 2.2, it follows from 2.3 that

$$
\begin{aligned}
A^{\star} X \Lambda^{2}+Q X \Lambda+A X & =\left(U^{\star} V\right) X \Lambda^{2}+\left(U^{\star} U+V^{\star} V\right) X \Lambda+\left(V^{\star} U\right) X \\
& =U^{\star}(V X \Lambda+U X) \Lambda+V^{\star}(V X \Lambda+U X)=0 .
\end{aligned}
$$

By this construction, we have the following factorization for $P(\lambda)$ :

$$
P(\lambda)=\lambda^{2} A^{\star}+\lambda Q+A=\left(\lambda U^{\star}+V^{\star}\right)(\lambda V+U) .
$$


However, it is not clear whether $P(\lambda)$ is singular for any $\lambda \in \mathbb{C}$, i.e., $\operatorname{det}(P(\lambda)) \equiv 0$, $\forall \lambda \in \mathbb{C}$. The condition $p \leq n$ plays a pivotal role in Problem PIEP. If $p>n$, then we can see from $m=2 n-p$ that $m<n$. Since $U, V \in \mathbb{C}^{m \times n}, \operatorname{rank}(\lambda V+U) \leq m<n$. It follows from (2.4) that $\operatorname{rank}(P(\lambda))<n$ and $\operatorname{det}(P(\lambda)) \equiv 0$ for all $\lambda \in \mathbb{C}$, i.e., $P(\lambda)$ is always singular. Hence, we always assume that $p \leq n$. Next, we claim that the assumption that $X$ has full column rank is sufficient for the regularity of $P(\lambda)$.

Theorem 2.2. Suppose for given matrix pair $(\Lambda, X) \in \mathbb{C}^{p \times p} \times \mathbb{C}^{n \times p}(p \leq n)$ that $X$ has full column rank. Then there are two $n \times n$ matrices $\widetilde{U}, \widetilde{V}$ over $\mathbb{C}$ with $\widetilde{V}$ nonsingular, such that $(\Lambda, X)$ is an eigen-matrix pair of the regular quadratic palindromic polynomial $P(\lambda)=\lambda A^{\star}+\lambda Q+A$ with coefficients given by

$$
A=\widetilde{V}^{\star} \widetilde{U}, \quad Q=\widetilde{U}^{\star} \widetilde{U}+\widetilde{V}^{\star} \widetilde{V},
$$

where $\widetilde{U}, \widetilde{V}$ satisfy

$$
\left(\begin{array}{ll}
X^{\star} & \Lambda^{\star} X^{\star}
\end{array}\right)\left(\begin{array}{l}
\widetilde{U}^{\star} \\
\widetilde{V}^{\star}
\end{array}\right)=0 .
$$

Proof. Let $U, V \in \mathbb{C}^{m \times n}$ be matrices defined by 2.2 . Since $X$ has full column rank, $m=2 n-p$. Then by Theorem 2.1 we have

$$
A^{\star} X \Lambda^{2}+Q X \Lambda+A X=0
$$

where $A=V^{\star} U, Q=U^{\star} U+V^{\star} V$. Similar to the proof of Theorem 2.2 in [10], we can prove that $V$ is of full column rank. Otherwise, there exists an unitary matrix $G \in \mathbb{C}^{m \times m}$ such that

$$
V^{\star} G=\left(\begin{array}{cc}
V_{1}^{\star} & 0_{n \times m_{2}}
\end{array}\right)
$$

where $V_{1}^{\star} \in \mathbb{C}^{n \times m_{1}}$ is of full column rank and $m_{1}<n, m=m_{1}+m_{2}$. Partition the matrix $U^{\star} G$ according to 2.7 into

$$
U^{\star} G=\left(\begin{array}{cc}
U_{1}^{\star} & U_{2}^{\star}
\end{array}\right)
$$

where $U_{1}^{\star} \in \mathbb{C}^{n \times m_{1}}, U_{2}^{\star} \in \mathbb{C}^{n \times m_{2}}$. We can obtain from $(2.2)$ that $X^{\star} U_{2}^{\star}=0$, where the columns of $U_{2}^{\star}$ are necessarily linearly independent by construction. It follows that

$$
n-p \geq m_{2}>m-n,
$$

which is contradict to the fact that $m=2 n-p$. Therefore, we have $\operatorname{rank}(V)=n, m_{1}=n$ and $V_{1} \in \mathbb{C}^{n \times n}$ is nonsingular. 
Now, we consider the structure of the basis of $\mathcal{N}(\Omega)$. By $(2.2)$, we know that the basis of $\mathcal{N}(\Omega)$ has the following form

$$
\left(\begin{array}{c}
U^{\star} \\
V^{\star}
\end{array}\right)=\left(\begin{array}{cc}
U_{1}^{\star} & U_{2}^{\star} \\
V_{1}^{\star} & 0_{m-n}
\end{array}\right),
$$

and hence $U_{1}, V_{1} \in \mathbb{C}^{n \times n}, U_{2} \in \mathbb{C}^{n \times(m-n)}$ satisfy

$$
U_{1} X+V_{1} X \Lambda=0, \quad U_{2} X=0 .
$$

By (2.6), 2.7) and (2.8), we have

$$
A=V^{\star} U=V_{1}^{\star} U_{1}, \quad Q=U^{\star} U+V^{\star} V=U_{1}^{\star} U_{1}+U_{2}^{\star} U_{2}+V_{1}^{\star} V_{1} .
$$

It follows that

$$
\begin{aligned}
& U_{1}^{\star} V_{1} X \Lambda^{2}+\left(U_{1}^{\star} U_{1}+V_{1}^{\star} V_{1}\right) X \Lambda+V_{1}^{\star} U_{1} X \\
= & U_{1}^{\star} V_{1} X \Lambda^{2}+\left(U_{1}^{\star} U_{1}+U_{2}^{\star} U_{2}+V_{1}^{\star} V_{1}\right) X \Lambda+V_{1}^{\star} U_{1} X \\
= & A^{\star} X \Lambda^{2}+Q X \Lambda+A X \\
= & 0,
\end{aligned}
$$

which implies that $(\Lambda, X)$ is an eigen-matrix pair of the quadratic palindromic polynomial $\widetilde{P}(\lambda)=\lambda^{2} \widetilde{A^{\star}}+\lambda \widetilde{Q}+\widetilde{A}$ with $\widetilde{A}=V_{1}^{\star} U_{1}$ and $\widetilde{Q}=U_{1}^{\star} U_{1}+V_{1}^{\star} V_{1}$.

Next, we prove that $\widetilde{P}(\lambda)$ is regular. By the generalized Schur decomposition [13], there exist two $n \times n$ unitary matrices $P$ and $Z$ such that

$$
U_{1}=P S Z^{\star}, \quad V_{1}=P T Z^{\star},
$$

where $S=\left(s_{i j}\right)$ and $T=\left(t_{i j}\right)$ are upper triangular. Then

$$
\widetilde{P}(\lambda)=\lambda^{2} \widetilde{A}^{\star}+\lambda \widetilde{Q}+\widetilde{A}=Z\left(\lambda S^{\star}+T^{\star}\right)(\lambda T+S) Z^{\star},
$$

which implies that

$$
\operatorname{det}(\widetilde{P}(\lambda))=\prod_{i=1}^{n}\left(\lambda s_{i i}^{\star}+t_{i i}^{\star}\right)\left(\lambda t_{i i}+s_{i i}\right) .
$$

Since $V_{1}$ is nonsingular, $t_{i i} \neq 0, i=1, \ldots, n$. It follows from 2.9 that $\operatorname{det}(\widetilde{P}(\lambda))$ is not identically zero for all $\lambda \in \mathbb{C}$, i.e., $\widetilde{P}(\lambda)$ is regular.

Corollary 2.3. Let $P(\lambda)$ be the quadratic palindromic polynomial that

$$
P(\lambda):=\lambda^{2} A^{\star}+\lambda Q+A \in \mathbb{C}^{n \times n},
$$

where $A=V^{\star} U, Q=U^{\star} U+V^{\star} V$ and $U, V \in \mathbb{C}^{n \times n}$. If $U$ or $V$ is nonsingular, then $P(\lambda)$ is regular. 
Theorem 2.4. Let $(\Lambda, X) \in \mathbb{C}^{p \times p} \times \mathbb{C}^{n \times p}(p \leq n)$ and $X$ be of full column rank. For any nonsingular matrix $V \in \mathbb{C}^{n \times n}$, there exits a regular quadratic palindromic polynomial $P(\lambda)$ defined by 2.5 with $(\Lambda, X)$ as its eigen-matrix pair.

Proof. Let $V \in \mathbb{C}^{n \times n}$ be any nonsingular matrix. We consider the following equation

$$
U X=-V X \Lambda
$$

where $U \in \mathbb{C}^{n \times n}$. Since $X$ is of full column rank, there exits an unitary matrix $Q \in \mathbb{C}^{n \times n}$ such that

$$
X=Q\left(\begin{array}{l}
R \\
0
\end{array}\right) \text {, }
$$

where $R \in \mathbb{C}^{p \times p}$ is nonsingular. Partition $U Q$ according to 2.11 into

$$
U Q=\left(Y_{1}, Y_{2}\right) \in \mathbb{C}^{n \times n}
$$

where $Y_{1} \in \mathbb{C}^{n \times p}$. Substituting 2.11) into 2.10, we get

$$
\widehat{U}=\left(-V X \Lambda R^{-1}, Y_{2}\right) Q^{\star} \in \mathbb{C}^{n \times n},
$$

where $Y_{2} \in \mathbb{C}^{n \times(n-p)}$ is arbitrary, which is the general solution of 2.10 . By Theorems 2.1 and 2.2, we know that Theorem 2.4 holds.

We have shown in the preceding part that how to define the coefficients so that the corresponding quadratic palindromic polynomial $P(\lambda)$ is regular and possesses a prescribed set of $p$ eigenvalues and eigenvectors. Problem PIEP is solved via construction and it follows from Theorem 2.4 that the solution of problem with the given eigen-matrix pair $(\Lambda, X)$ is not unique. Now, we consider the eigenstructure of $P(\lambda)$.

Theorem 2.5. Let $P(\lambda)=\lambda^{2} A^{\star}+\lambda Q+A$ be the regular quadratic palindromic polynomial with $A=V^{\star} U, Q=U^{\star} U+V^{\star} V$, where $U, V \in \mathbb{C}^{n \times n}$ and $V$ is nonsingular. Assume that $\operatorname{rank}(U)=r$, then

(1) If $r=n$, then $P(\lambda)$ has $n$ nonzero eigenvalue pairs $\left(\lambda_{j}, 1 / \lambda_{j}^{\star}\right), j=1, \ldots, n$.

(2) If $r<n$, then $P(\lambda)$ has $r$ nonzero eigenvalue pairs $\left(\lambda_{j}, 1 / \lambda_{j}^{\star}\right), j=1, \ldots, r$, and the remaining $2(n-r)$ eigenvalues of $P(\lambda)$ are $n-r$ eigenvalues pairs $(0, \infty)$.

Proof. By the generalized Schur decomposition [13], there exist two $n \times n$ unitary matrices $P$ and $Z$ such that

$$
U=P S Z^{\star}, \quad V=P T Z^{\star},
$$


where $S=\left(s_{i j}\right) \in \mathbb{C}^{n \times n}$ and $T=\left(t_{i j}\right) \in \mathbb{C}^{n \times n}$ are upper triangular. Since $V$ is nonsingular, $t_{i i} \neq 0, i=1, \ldots, n$. Then

$$
P(\lambda)=\lambda^{2} A^{\star}+\lambda Q+A=Z\left(\lambda S^{\star}+T^{\star}\right)(\lambda T+S) Z^{\star}
$$

which implies that

$$
\operatorname{det}(P(\lambda))=\prod_{i=1}^{n}\left(\lambda s_{i i}^{\star}+t_{i i}^{\star}\right)\left(\lambda t_{i i}+s_{i i}\right) .
$$

(1) If $r=n$, then $U$ is nonsingular, i.e., $s_{i i} \neq 0, i=1, \ldots, n$. By 2.12), it is easy to see that $P(\lambda)$ has eigenvalue pairs $\left(\lambda_{i}, 1 / \lambda_{i}^{\star}\right)$ with $\lambda_{i}=-s_{i i} / t_{i i}, i=1, \ldots, n$.

(2) If $r<n$, then $U$ is singular. Without loss of generality, we can assume that $s_{i i} \neq 0$, $i=1, \ldots, r$ and $s_{i i}=0, i=r+1, \ldots, n$. Then 2.12 can be written as

$$
\operatorname{det}(P(\lambda))=\prod_{i=1}^{r}\left(\lambda s_{i i}^{\star}+t_{i i}^{\star}\right)\left(\lambda t_{i i}+s_{i i}\right) \prod_{i=r+1}^{n} \lambda t_{i i}^{\star} t_{i i},
$$

which implies that $P(\lambda)$ has eigenvalue pairs $\left(\lambda_{j}, 1 / \lambda_{j}^{\star}\right), j=1, \ldots, r$ with $\lambda_{j}=-s_{j j} / t_{j j}$. The remaining $2(n-r)$ eigenvalues of $P(\lambda)$ are $n-r$ eigenvalue pairs $(0, \infty)$.

\section{Solutions of Problem PIEP for $1 \leq p<2 n$}

We have seen in the preceding section that there always exists a regular quadratic palindromic polynomial which can have prescribed $p(p \leq n)$ eigenvalues and $p$ linearly independent eigenvectors. A natural problem is whether the Problem PIEP can be solved for general $p(1 \leq p \leq 2 n)$. In [9], Chu constructed a symmetric quadratic polynomial $\lambda^{2} M+\lambda C+K$ with prescribed $p$ eigenpairs $(\lambda, x)$, where $p$ can be any integer between 1 and $2 n$. In this section, we give the general solution of Problem PIEP for any $p(1 \leq p \leq 2 n)$ prescribed eigen-matrix pair $(\Lambda, X) \in \mathbb{C}^{p \times p} \times \mathbb{C}^{n \times p}$.

Without loss of generality, we adopt the following notations and make some assumptions throughout this section.

A2 Assume that $\Lambda$ is nonsingular and all eigenvalues of $\Lambda$ are distinct.

A3 Assume that $\Lambda$ has the following form

$$
\Lambda=\operatorname{diag}\left(\lambda_{1}, \ldots, \lambda_{p}\right) \in \mathbb{C}^{p \times p}
$$

where $\lambda_{2 k-1} \lambda_{2 k}^{\star}=1$ for $k=1, \ldots, q ; \lambda_{i} \lambda_{j}^{\star} \neq 1$ for $i=1, \ldots, p, j=2 q+1, \ldots, p$, and $\Lambda$ has no exceptional eigenvalue, i.e., $\lambda \lambda^{\star} \neq 1$ for any eigenvalue $\lambda$ of $\Lambda$. 
Consider the algebraic system

$$
A^{\star} X \Lambda^{2}+Q X \Lambda+A X=0_{n \times p}
$$

where $A, Q \in \mathbb{C}^{n \times n}$ with $Q^{\star}=Q$. First, we analyze the necessary condition for the quadratic palindromic polynomial $P(\lambda)=\lambda^{2} A^{\star}+\lambda Q+A$ that has eigen-matrix pair $(\Lambda, X)$, i.e., 3.2 holds. Define

$$
\mathbb{S}_{\Lambda}=\left\{T \in \mathbb{C}^{p \times p} \mid T \Lambda^{-1}+T^{\star} \Lambda=\Lambda^{-\star} T^{\star}+\Lambda^{\star} T\right\}
$$

Note that $Q^{\star}=Q$, then $(3.2)$ can be rewritten as

$$
\Lambda^{\star 2} X^{\star} A+\Lambda^{\star} X^{\star} Q+X^{\star} A^{\star}=0 .
$$

Denote

$$
S:=X^{\star} A X \in \mathbb{C}^{p \times p} .
$$

It is easy to see that for any matrix pair $(A, Q)$ satisfying 3.4 must satisfy

$$
\Lambda^{\star} X^{\star} Q X=-S^{\star}-\Lambda^{\star 2} S
$$

Since $\Lambda$ is nonsingular, we have

$$
Q^{\star}=Q \quad \Longleftrightarrow \quad S \in \mathbb{S}_{\Lambda}
$$

We now consider the structure of the parameter matrix $S:=\left(s_{i j}\right)_{p \times p} \in \mathbb{S}_{\Lambda}$. It follows from $(3.3)$ that

$$
\lambda_{i}^{\star}\left(1-\lambda_{i}^{\star} \lambda_{j}\right) s_{i j}=\lambda_{j}\left(1-\lambda_{i}^{\star} \lambda_{j}\right) s_{j i}^{\star}, \quad i, j=1, \ldots, p .
$$

For $i=j$, since $\Lambda$ has no exceptional eigenvalue, $\lambda_{i} \lambda_{i}^{\star} \neq 1, i=1, \ldots, p$, which implies that

$$
s_{i i}=\lambda_{i} \lambda_{i}^{-\star} s_{i i}^{\star}, \quad i=1, \ldots, p .
$$

If $\lambda_{i} \in \mathbb{R}$, then $s_{i i} \in \mathbb{R}$ is arbitrary. If $\lambda \notin \mathbb{R}$, it follows from 3.8 that $s_{i i}=\frac{a}{\operatorname{Im}\left(\lambda_{i}\right)} \lambda_{i}$, where $a \in \mathbb{R}$ is arbitrary and $\operatorname{Im}\left(\lambda_{i}\right)$ is the imaginary part of $\lambda_{i}$. For $i \neq j$, if $\lambda_{i}^{\star} \lambda_{j}=1$, then (3.7) holds for arbitrary $s_{i j}, s_{j i} \in \mathbb{C}$; if $\lambda_{i}^{\star} \lambda_{j} \neq 1$, then $s_{i j}=\lambda_{i}^{-\star} \lambda_{j} s_{j i}^{\star}$. Since the eigenvalues of $\Lambda$ are distinct, we can see from the discussion above that the freedom (complex degree) of matrix $S$ is $p(p+1) / 2+q$.

Lemma 3.1. Let $\Lambda \in \mathbb{C}^{p \times p}$ be the matrix defined by 3.1) and $S=\left(s_{i j}\right) \in \mathbb{C}^{p \times p}$. If $S \in \mathbb{S}_{\Lambda}$, then the freedom (complex degree) of parameter matrix $S$ is $p(p+1) / 2+q$ and 
(1) $s_{i i}=\left\{\begin{array}{ll}a & \text { if } \lambda_{i} \in \mathbb{R}, \\ a \lambda_{i} / \operatorname{Im}\left(\lambda_{i}\right) & \text { if } \lambda_{i} \notin \mathbb{R},\end{array} \quad \forall a \in \mathbb{R}, i=1, \ldots, p ;\right.$

(2) $s_{i j}, s_{j i} \in \mathbb{C}$ are arbitrary, $i=2 k-1, j=2 k, k=1, \ldots, q$;

(3) $s_{i j}=\lambda_{i}^{-\star} \lambda_{j} s_{j i}^{\star}, \forall s_{j i} \in \mathbb{C}, i=1, \ldots, p, j=2 q+1, \ldots, p$.

The conditions given by Lemma 3.1 can be used to construct the solution $(A, Q)$ of Problem PIEP in terms of matrix $S \in \mathbb{S}_{\Lambda}$. For simplicity, we divide our discussion into three cases.

\subsection{The case $p=n$}

For a given matrix $S \in \mathbb{S}_{\Lambda}$, we need to see how $A$ and $Q$ can be determined from the equations (3.5) and (3.6). Since $X \in \mathbb{C}^{n \times n}$ is nonsingular and $Q^{\star}=Q$, (3.5) and (3.6) can be rewritten as

$$
A=X^{-\star} S X^{-1}, \quad Q=X^{-\star}\left(S \Lambda^{-1}+S^{\star} \Lambda\right) X^{-1}
$$

Clearly, $A$ and $Q$ are totally determined by $S$ which has $n(n+1) / 2+q$ free parameters. Thus, we have the following result.

Theorem 3.2. Let $(\Lambda, X) \in \mathbb{C}^{n \times n} \times \mathbb{C}^{n \times n}$. For any nonzero matrix $S \in \mathbb{S}_{\Lambda}$, there exists a quadratic palindromic polynomial $P(\lambda)=\lambda^{2} A^{\star}+\lambda Q+A$ with coefficients $A$ and $Q$ defined by (3.9), which has the matrix pair $(\Lambda, X)$ as part of its eigeninformation.

Corollary 3.3. With $(A, Q)$ being defined in Theorem 3.2, the corresponding quadratic palindromic polynomial $P(\lambda)$ can be factorized as follows:

$$
\lambda^{2} A^{\star}+\lambda Q+A=X^{-\star}\left(\lambda S^{\star}-S \Lambda^{-1}\right)(\lambda I-\Lambda) X^{-1},
$$

which shows that $P(\lambda)$ shares all the eigenvalues of $\Lambda$. Moreover, if $S \in \mathbb{S}_{\Lambda}$ is nonsingular, then the resulting polynomial $P(\lambda)$ is regular.

Proof. It follows from 3.9 that

$$
\begin{aligned}
\lambda^{2} A^{\star}+\lambda Q+A & =X^{-\star}\left[\lambda^{2} S^{\star}-\lambda\left(S \Lambda^{-1}+S^{\star} \Lambda\right)+S\right] X^{-1} \\
& =X^{-\star}\left[S^{\star}\left(\lambda^{2} I-\lambda \Lambda\right)-S\left(\lambda \Lambda^{-1}-I\right)\right] X^{-1} \\
& =X^{-\star}\left(\lambda S^{\star}-S \Lambda^{-1}\right)(\lambda I-\Lambda) X^{-1} .
\end{aligned}
$$

It is easy to see from Lemma 3.1 that we can choose $S \in \mathbb{S}_{\Lambda}$ to be nonsingular, and then $A=X^{-\star} S X^{-1}$ is nonsingular which implies that $P(\lambda)=\lambda^{2} A^{\star}+\lambda Q+A$ is regular. 
Remark 3.4. Since $S \in \mathbb{S}_{\Lambda}$, the coefficient matrix $Q$ of the resulting polynomial $P(\lambda)$ can be also written as

$$
Q=-X^{-\star}\left(\Lambda^{-\star} S^{\star}+\Lambda^{\star} S\right) X^{-1} \text {. }
$$

Similar to the proof of Corollary 3.3 , the solution $P(\lambda)$ can be also factorized as

$$
\lambda^{2} A^{\star}+\lambda Q+A=X^{-\star}\left(\lambda I-\Lambda^{-\star}\right)\left(\lambda T^{\star}-\Lambda^{-\star} T\right) X^{-1},
$$

which shows that $P(\lambda)$ shares all the eigenvalues of $\Lambda^{-\star}$. This is consistent with the property "symplectic spectral symmetry" of the eigenvalues of quadratic palindromic polynomial.

\subsection{The case $p<n$}

Let the QR-decomposition of $X$ be given by

$$
X=P\left(\begin{array}{l}
R \\
0
\end{array}\right)
$$

where $R \in \mathbb{C}^{p \times p}$ is nonsingular and $P \in \mathbb{C}^{n \times n}$ is an unitary matrix. Then (3.2) is equivalent to

$$
P^{\star} A^{\star} P\left(\begin{array}{l}
R \\
0
\end{array}\right) \Lambda^{2}+P^{\star} Q P\left(\begin{array}{l}
R \\
0
\end{array}\right) \Lambda+P^{\star} A P=0 .
$$

Partition $P^{\star} A P$ and $P^{\star} Q P$ according to 3.10 into

$$
P^{\star} A P=\left(\begin{array}{cc}
A_{11} & A_{12} \\
A_{21} & A_{22}
\end{array}\right), \quad P^{\star} Q P=\left(\begin{array}{ll}
Q_{11} & Q_{12} \\
Q_{21} & Q_{22}
\end{array}\right),
$$

where $A_{11}, Q_{11} \in \mathbb{C}^{p \times p}$ and $Q_{11}^{\star}=Q_{11}$. Denote $\Gamma=R \Lambda R^{-1}$. Hence, (3.11) can be rewritten as

$$
\left(\begin{array}{cc}
A_{11}^{\star} & A_{21}^{\star} \\
A_{12}^{\star} & A_{22}^{\star}
\end{array}\right)\left(\begin{array}{c}
R \Lambda^{2} \\
0
\end{array}\right)+\left(\begin{array}{ll}
Q_{11} & Q_{12} \\
Q_{21} & Q_{22}
\end{array}\right)\left(\begin{array}{c}
R \Lambda \\
0
\end{array}\right)+\left(\begin{array}{ll}
A_{11} & A_{12} \\
A_{21} & A_{22}
\end{array}\right)\left(\begin{array}{l}
R \\
0
\end{array}\right)=0,
$$

which implies that

$$
A_{11}^{\star} \Gamma^{2}+Q_{11} \Gamma+A_{11}=0
$$

and

$$
A_{12}^{\star} \Gamma^{2}+Q_{21} \Gamma+A_{21}=0 .
$$


Since $\Lambda$ is nonsingular, it follows from 3.12 and 3.13 that

$$
\begin{aligned}
& Q_{11}=-\left(A_{11}^{\star} \Gamma+A_{11} \Gamma^{-1}\right), \\
& Q_{21}=-\left(A_{12}^{\star} \Gamma+A_{21} \Gamma^{-1}\right) .
\end{aligned}
$$

Since $Q^{\star}=Q$, we obtain from (3.14) that

$$
A_{11}^{\star} \Gamma+A_{11} \Gamma^{-1}=\Gamma^{\star} A_{11}+\Gamma^{-\star} A_{11}^{\star} .
$$

Substituting $\Gamma=R \Lambda R^{-1}$ into above equation, we have

$$
\left(R^{\star} A_{11}^{\star} R\right) \Lambda+\left(R^{\star} A_{11} R\right) \Lambda^{-1}=\Lambda^{\star}\left(R^{\star} A_{11} R\right)+\Lambda^{-\star}\left(R^{\star} A_{11}^{\star} R\right) .
$$

Denote $T=R^{\star} A_{11} R$. Then, we can obtain from 3.15 that

$$
Q_{11}^{\star}=Q_{11} \Longleftrightarrow T \in \mathbb{S}_{\Lambda}
$$

Therefore, we have proved the following result.

Theorem 3.5. Let the $Q R$-decomposition of $X$ be given by 3.10 and $\Gamma=R \Lambda R^{-1}$. Then the general solution to Problem PIEP with $p \leq n$ is given by

$$
A=P\left(\begin{array}{ll}
A_{11} & A_{12} \\
A_{21} & A_{22}
\end{array}\right) P^{\star}, \quad Q=P\left(\begin{array}{ll}
Q_{11} & Q_{12} \\
Q_{21} & Q_{22}
\end{array}\right) P^{\star},
$$

where

(i) $A_{12} \in \mathbb{C}^{p \times(n-p)}, A_{21} \in \mathbb{C}^{(n-p) \times p}$ and $A_{22} \in \mathbb{C}^{(n-p) \times(n-p)}$ are arbitrary,

(ii) $Q_{22}=Q_{22}^{\star} \in \mathbb{C}^{(n-p) \times(n-p)}$ is arbitrary,

(iii) $A_{11}=R^{-\star} T R^{-1} \in \mathbb{C}^{p \times p}$ for any nonzero matrix $T \in \mathbb{S}_{\Lambda}$,

(iv) $Q_{11}=-\left(A_{11}^{\star} \Gamma+A_{11} \Gamma^{-1}\right)$,

(v) $Q_{21}=Q_{12}^{\star}=-\left(A_{12}^{\star} \Gamma+A_{21} \Gamma^{-1}\right)$.

Corollary 3.6. In the case $p<n$, the corresponding quadratic palindromic polynomial $P(\lambda)$ can be factorized as

$$
\begin{aligned}
P(\lambda) & =\lambda^{2} A^{\star}+\lambda Q+A \\
& =\left(\begin{array}{cc}
R^{-\star}\left(\lambda T^{\star}-T \Lambda^{-1}\right) & R^{-\star}\left(\lambda I-\Lambda^{-\star}\right)\left(\lambda R^{\star} A_{21}^{\star}-\Lambda^{\star} R^{\star} A_{12}\right) \\
\left(\lambda A_{12}^{\star} R-A_{21} R \Lambda^{-1}\right) & \lambda^{2} A_{22}^{\star}+\lambda Q_{22}+A_{22}
\end{array}\right)\left(\begin{array}{cc}
(\lambda I-\Lambda) R^{-1} & 0 \\
0 & I
\end{array}\right) \\
& =\left(\begin{array}{cc}
R^{-\star}\left(\lambda I-\Lambda^{-\star}\right) & 0 \\
0 & I
\end{array}\right)\left(\begin{array}{cc}
\left(\lambda T^{\star}-\Lambda^{-\star} T\right) R^{-1} & \left(\lambda R^{\star} A_{21}^{\star}-\Lambda^{\star} R^{\star} A_{12}\right) \\
\left(\lambda A_{12}^{\star} R-A_{21} R \Lambda^{-1}\right)(\lambda I-\Lambda) R^{-1} & \lambda^{2} A_{22}^{\star}+\lambda Q_{22}+A_{22}
\end{array}\right)
\end{aligned}
$$

which shows that the solution $P(\lambda)$ shares all the eigenvalues of $\Lambda$ and $\Lambda^{-\star}$. 
Proof. Similar to the proof of Corollary 3.3, we can obtain from (iii) and (iv) of Theorem 3.5 that

$$
\begin{aligned}
\lambda^{2} A_{11}^{\star}+\lambda Q_{11}+A_{11} & =R^{-\star}\left(\lambda T^{\star}-T \Lambda^{-1}\right)(\lambda I-\Lambda) R^{-1} \\
& =R^{-\star}\left(\lambda I-\Lambda^{-\star}\right)\left(\lambda T^{\star}-\Lambda^{-\star} T\right) R^{-1}
\end{aligned}
$$

We can see from Theorem 3.5(v) that

$$
\lambda^{2} A_{21}^{\star}+\lambda Q_{12}+A_{12}=R^{-\star}\left(\lambda I-\Lambda^{-\star}\right)\left(\lambda R^{\star} A_{21}^{\star}-\Lambda^{\star} R^{\star} A_{12}\right),
$$

and

$$
\lambda^{2} A_{12}^{\star}+\lambda Q_{21}+A_{21}=\left(\lambda A_{12}^{\star} R-A_{21} R \Lambda^{-1}\right)(\lambda I-\Lambda) R^{-1}
$$

It follows from (3.17), 3.18) and 3.19 that 3.16 holds.

\subsection{The case $p>n$}

Note that the eigenvalue of quadratic palindromic polynomial has the property "symplectic spectral symmetry", i.e., every eigenvalues occur in pairs $\left(\lambda, 1 / \lambda^{\star}\right)$. Since $p>n$, it is easy to verify that the number of the prescribed eigenvalues which occur in pairs can not be given arbitrarily. By the symplectic property of eigenvalues, we can see that the numbers $p, n, q$ of 3.1 must satisfy $p-2 q \leq 2 n-p$, i.e.,

$$
p-n \leq q<n
$$

First, we can see from 3.5 and $(3.6)$ that

$$
S=X^{\star} A X
$$

and

$$
X^{\star} Q X=-\Lambda^{-\star} S^{\star}-\Lambda^{\star} S
$$

where $S \in \mathbb{S}_{\Lambda}$. It follows from Assumption A1 that $X \in \mathbb{C}^{n \times p}$ is of full row rank, and then there exists an $p \times p$ permutation matrix $P$ such that

$$
X=\left(X_{1}, X_{2}\right) P
$$

where $X_{1} \in \mathbb{C}^{n \times n}$ is nonsingular and $X_{2} \in \mathbb{C}^{n \times(p-n)}$. By 3.2 and $(3.3)$, we have $S \in \mathbb{S}_{\Lambda}$ if and only if $P^{\star} S P \in \mathbb{S}_{P^{\star} \Lambda P}$, and

$$
A^{\star} X \Lambda^{2}+Q X \Lambda+A X=0
$$


if and only if

$$
A^{\star} X P\left(P^{\star} \Lambda P\right)^{2}+Q X P\left(P^{\star} \Lambda P\right)+A X P=0 .
$$

Since $P$ is a permutation matrix, it is easy to see that $P^{\star} \Lambda P$ is also diagonal and have the same eigenvalues with $\Lambda$. Thus, without loss of generality, we can assume that $P$ is an identity matrix. Then we can see from 3.21 that

$$
S=\left(\begin{array}{ll}
S_{11} & S_{12} \\
S_{21} & S_{22}
\end{array}\right)=\left(\begin{array}{cc}
X_{1}^{\star} A X_{1} & X_{1}^{\star} A X_{2} \\
X_{2}^{\star} A X_{1} & X_{2}^{\star} A X_{2}
\end{array}\right),
$$

where $S_{i j}, i, j=1,2$, are blocks with appropriate sizes. Partition $\Lambda$ according to 3.23 into

$$
\Lambda=\left(\begin{array}{cc}
\Lambda_{1} & 0 \\
0 & \Lambda_{2}
\end{array}\right)
$$

where $\Lambda_{1} \in \mathbb{C}^{n \times n}$. The relationship 3.23 suggests that we may choose $S_{11} \in \mathbb{S}_{\Lambda_{1}}$ such that

$$
A=X_{1}^{-\star} S_{11} X_{1}^{-1}
$$

Substituting (3.24) into (3.23), we have

$$
S=\left(\begin{array}{cc}
S_{11} & S_{11} X_{1}^{-1} X_{2} \\
X_{2}^{\star} X_{1}^{-\star} S_{11} & X_{2}^{\star} X_{1}^{-\star} S_{11} X_{1}^{-1} X_{2}
\end{array}\right) .
$$

In order to obtain the matrix $Q$ by $(3.22)$, we see from $Q^{\star}=Q$ that the matrix $S$ of form (3.25) must satisfy

$$
S^{\star} \Lambda+S \Lambda^{-1}=\Lambda^{\star} S+\Lambda^{-\star} S^{\star}
$$

Let $Y:=X_{1}^{-1} X_{2}$. Substituting $S$ into both side of equation 3.26 , then the critical condition 3.26 can be expressed as the following equations:

$$
\begin{aligned}
S_{11}^{\star} \Lambda_{1}+S_{11} \Lambda_{1}^{-1} & =\Lambda_{1}^{\star} S_{11}+\Lambda_{1}^{-\star} S_{11}^{\star}, \\
S_{11}^{\star} Y \Lambda_{2}+S_{11} Y \Lambda_{2}^{-1} & =\left(\Lambda_{1}^{\star} S_{11}+\Lambda_{1}^{-\star} S_{11}^{\star}\right) Y, \\
Y^{\star}\left(S_{11}^{\star} \Lambda_{1}+S_{11} \Lambda_{1}^{-1}\right) & =\Lambda_{2}^{\star} Y^{\star} S_{11}+\Lambda_{2}^{-\star} Y^{\star} S_{11}^{\star}, \\
Y^{\star}\left(S_{11}^{\star} Y \Lambda_{2}+S_{11} Y \Lambda_{2}^{-1}\right) & =\left(\Lambda_{2}^{\star} Y^{\star} S_{11}+\Lambda_{2}^{-\star} Y^{\star} S_{11}^{\star}\right) Y .
\end{aligned}
$$

It is easy to verify that 3.28 holds if and only if 3.29 holds. Post-multiplying (3.27) by $Y$ and subtracting 3.28 , we obtain that

$$
S_{11}^{\star} Y \Lambda_{2}+S_{11} Y \Lambda_{2}^{-1}=S_{11}^{\star} \Lambda_{1} Y+S_{11} \Lambda_{1}^{-1} Y
$$


We can see from 3.29 and 3.31 that

$$
\begin{aligned}
Y^{\star}\left(S_{11}^{\star} Y \Lambda_{2}+S_{11} X_{1}^{-1} Y\right) & =Y^{\star}\left(S_{11}^{\star} \Lambda_{1} Y+S_{11} \Lambda_{1}^{-1} Y\right) \\
& =\left(Y^{\star} S_{11}^{\star} \Lambda_{1} Y+Y^{\star} S_{11} \Lambda_{1}^{-1}\right) Y \\
& =\left(\Lambda_{2}^{\star} Y^{\star} S_{11}+\Lambda_{2}^{-\star} Y^{\star} S_{11}^{\star}\right) Y,
\end{aligned}
$$

which is precisely (3.30). Therefore, once (3.27) and (3.28) are solved by some matrix in $\mathbb{S}_{\Lambda_{1}}$, we can construct the matrix $Q$. Since

$$
X^{\star} Q X=\left(\begin{array}{cc}
X_{1}^{\star} Q X_{1} & X_{1}^{\star} Q X_{2} \\
X_{2}^{\star} Q X_{1} & X_{2}^{\star} Q X_{2}
\end{array}\right),
$$

according to (3.24) and (3.22), we may choose

$$
Q=-X_{1}^{\star}\left(S_{11}^{\star} \Lambda_{1}+S_{11} \Lambda_{1}^{-1}\right) X_{1}^{-1} .
$$

Now, we prove that $(\Lambda, X)$ is an eigenpair of the quadratic palindromic polynomial with coefficients $A$ and $Q$ defined by (3.24) and (3.32), respectively. We can see from (3.24), (3.31) and (3.32) that

$$
\begin{aligned}
& A^{\star} X \Lambda^{2}+Q X \Lambda+A X \\
= & X_{1}^{-\star} S_{11}^{\star} X_{1}^{-1}\left[X_{1}, X_{2}\right]\left(\begin{array}{cc}
\Lambda_{1}^{2} & 0 \\
0 & \Lambda_{2}^{2}
\end{array}\right)-X_{1}^{-\star}\left(S_{11}^{\star} \Lambda_{1}+S_{11} \Lambda_{1}^{-1}\right) X_{1}^{-1}\left[X_{1}, X_{2}\right]\left(\begin{array}{cc}
\Lambda_{1} & 0 \\
0 & \Lambda_{2}
\end{array}\right) \\
& +X_{1}^{-\star} S_{11} X_{1}^{-1}\left[X_{1}, X_{2}\right] \\
= & X_{1}^{-\star} S_{11}^{\star}\left[\Lambda_{1}^{2}, Y \Lambda_{2}^{2}\right]-X_{1}^{-\star}\left(S_{11}^{\star} \Lambda_{1}+S_{11} \Lambda_{1}^{-1}\right)\left[\Lambda_{1}, Y \Lambda_{2}\right]+X_{1}^{-\star} S_{11}[I, Y] \\
= & X_{1}^{-\star}\left[S_{11}^{\star} \Lambda_{1}^{2}+S_{11}, S_{11}^{\star} Y \Lambda_{2}^{2}+S_{11} Y\right]-X_{1}^{-\star}\left[S_{11}^{\star} \Lambda_{1}^{2}+S_{11}, S_{11}^{\star} \Lambda_{1} Y \Lambda_{2}+S_{11} \Lambda_{1}^{-1} Y \Lambda_{2}\right] \\
= & X_{1}^{-\star}\left[0,\left(S_{11}^{\star} Y \Lambda_{2}+S_{11} Y \Lambda_{2}^{-1}\right)-\left(S_{11}^{\star} \Lambda_{1} Y+S_{11} \Lambda_{1}^{-1} Y\right)\right] \Lambda_{2} \\
= & 0 .
\end{aligned}
$$

The last "=" follows from 3.31).

What we have just proved is that if we can solve equations 3.27 and (3.28) by some matrix in $\mathbb{S}_{\Lambda_{1}}$, then $(3.29)$ and $(3.30)$ are automatically solved. In this case, we can construct a palindromic polynomial with coefficient matrices $A$ and $Q$ defined by (3.24) and (3.32), respectively, which has $(\Lambda, X)$ as its eigeninformation. Clearly, if the $n \times n$ matrix $S_{11}$ satisfies (3.27), then $S_{11} \in \mathbb{S}_{\Lambda_{1}}$. We know from Lemma 3.1 and 3.20 that there are $n(n+1) / 2+q$ free parameters of $S_{11} \in \mathbb{S}_{\Lambda_{1}}$. Since there are $n(p-n)$ linear equations imposed by (3.28), there remains totally only

$$
\frac{n(n+1)}{2}+q-n(p-n)=\frac{n(3 n+1)}{2}+q-n p
$$

degrees of freedom in $S_{11}$. Therefore, we have the following result. 
Theorem 3.7. Let $(\Lambda, X) \in \mathbb{C}^{p \times p} \times \mathbb{C}^{n \times p}$. For any $n \leq p \leq(3 n+1) / 2$, Problem PIEP is always solvable under the Assumptions A1-A3.

Proof. We can see from $(3.33)$ that if

$$
\frac{n(3 n+1)}{2}+q-n p>0
$$

i.e.,

$$
p<\frac{3 n+1}{2}+\frac{q}{n}
$$

then there exists nonzero matrix $S_{11} \in \mathbb{C}^{n \times n}$ which can solve the equations (3.27) and (3.28). And in this case, Problem PIEP is solvable. It follows from $q<n$ and 3.34 that $p \leq(3 n+1) / 2$.

Together with Theorems 3.2, 3.5 and 3.7, we have now established the following result.

Theorem 3.8. Given any integer $p(1 \leq p \leq(3 n+1) / 2)$, Problem PIEP is always solvable under the Assumptions A1-A3.

Remark 3.9. We can see from Theorem 3.8 that the maximal allowable number $p_{\max }$ of prescribed eigenpairs is given by

$$
p_{\max }= \begin{cases}3 k & \text { if } n=2 k, \\ 3 k+2 & \text { if } n=2 k+1 .\end{cases}
$$

\section{Numerical experiments}

In this section, we will present some examples to illustrate the methods of Sections 2 and 3 for solving Problem PIEP on two different types of palindromic polynomial as shown in (1.2) and 1.3 .

Example 4.1. The following are some examples illustrating the performance of Theorem 2.4 for solving Problem PIEP with $n=6, p=4$. Setting

$$
X_{1}=\left[\begin{array}{cccc}
i & 1 & 0 & 0 \\
1 & i & i & 0 \\
1 & 1 & 1 & i \\
1 & 1 & 1 & 1 \\
i & i & 1 & 1 \\
1 & i & 1 & i
\end{array}\right], \quad \Lambda_{1}= \begin{cases}\operatorname{diag}\left(2+i, \frac{1}{2-i}, 3+2 i, \frac{1}{3-2 i}\right) & \text { if } \star=*, \\
\operatorname{diag}\left(2+i, \frac{1}{2+i}, 3+2 i, \frac{1}{3+2 i}\right) & \text { if } \star=\top .\end{cases}
$$


Without loss of generality, we can assume that $V$ is an $6 \times 6$ identity matrix. By Theorem 2.4, we choose $Y_{2} \in \mathbb{C}^{6 \times 2}$ randomly and get an $*$-palindromic polynomial $P(\lambda)=$ $\lambda^{2} A^{*}+\lambda Q+A$ with

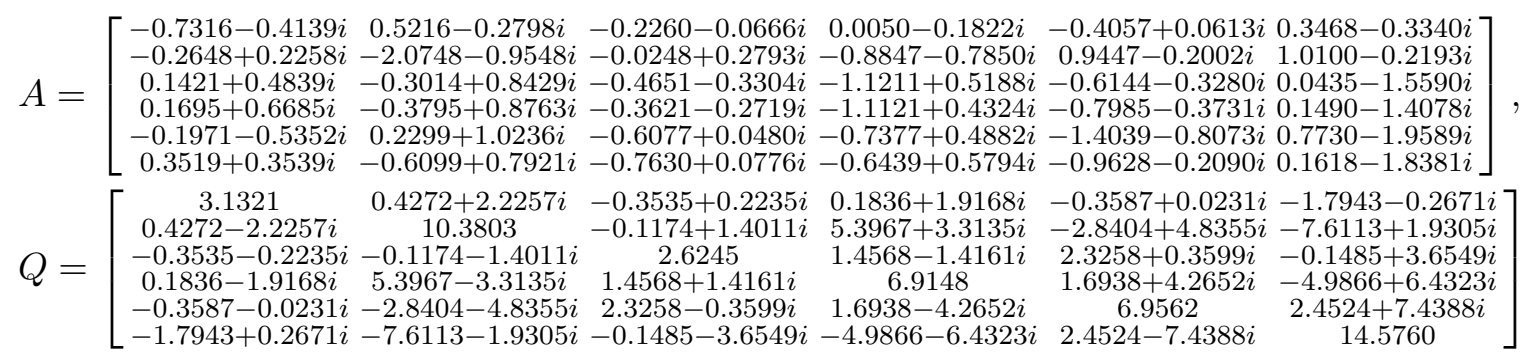

which satisfies

$$
\left\|Q-Q^{*}\right\|_{F}=2.6710 e-15, \quad\left\|A^{*} X_{1} \Lambda_{1}^{2}+Q X_{1} \Lambda_{1}+A X_{1}\right\|_{F}=4.3188 e-14,
$$

and also a $\top$-palindromic polynomial $P(\lambda)=\lambda^{2} A^{\top}+\lambda Q+A$ with

$$
\begin{aligned}
A= & {\left[\begin{array}{cccccc}
-0.7785-0.4539 i & 0.3972-0.2658 i & -0.5586+0.2565 i & 0.1551-0.1375 i & -0.4866+0.0718 i & 0.6243-0.5880 i \\
-0.3618+0.4348 i & -2.1927-0.7056 i & -0.1801+0.3032 i & -0.5272-0.9469 i & 0.7708-0.1039 i & 1.2309-0.0596 i \\
0.1040+0.5136 i & -0.4662+0.8271 i & -0.7283+0.0524 i & -1.0398+0.5050 i & -0.7751-0.3777 i & 0.3703-1.7135 i \\
0.1399+0.6368 i & -0.4457+0.9017 i & -0.4653-0.1228 i & -1.1845+0.5803 i & -0.8341-0.3467 i & 0.3856-1.6650 i \\
-0.2867-0.3869 i & 0.2268+1.2234 i & -0.4691-0.1529 i & -0.7576+0.4628 i & -1.4364-0.7264 i & 0.8865-1.8104 i \\
0.2197+0.3136 i & -0.5390+0.7448 i & -0.5942-0.0347 i & -1.0140+0.5069 i & -0.8056-0.3022 i & 0.1587-1.6312 i
\end{array}\right], } \\
Q= & {\left[\begin{array}{cccccc}
0.5855+1.0366 i & -0.3831-1.4264 i & 0.3507-0.7849 i & -0.4689-1.0508 i & 0.4437-0.0343 i & 0.4521+0.6232 i \\
-0.3831-1.4264 i & 2.6067+1.0602 i & 1.4961-2.3114 i & 0.0157-1.9201 i & 0.6428-3.5798 i & 3.4698+2.7290 i \\
0.3507-0.7849 i & 1.4961-2.3114 i & 2.4645-0.1732 i & 2.7302-0.7870 i & 2.1070+1.3535 i & -1.8087+4.5447 i \\
-0.4689-1.0508 i & 0.0157-1.9201 i & 2.7302-0.7870 i & 3.4100-3.1985 i & 4.0100-0.8854 i & 1.1326+6.3698 i \\
0.4437-0.0343 i & 0.6428-3.5798 i & 2.1070+1.3535 i & 4.0100-0.8854 i & 4.9417+3.5075 i & -4.3613+5.8228 i \\
0.4521+0.6232 i & 3.4698+2.7290 i & -1.8087+4.5447 i & 1.1326+6.3698 i & -4.3613+5.8228 i & -7.9937-7.1613 i
\end{array}\right] }
\end{aligned}
$$

which satisfies

$$
\left\|Q-Q^{\top}\right\|_{F}=2.6865 e-15, \quad\left\|A^{\top} X_{1} \Lambda_{1}^{2}+Q X_{1} \Lambda_{1}+A X_{1}\right\|_{F}=4.4740 e-14 .
$$

Example 4.2. The following are some examples illustrating the performance of Theorems 3.2 and 3.5 for solving Problem PIEP. We only concentrate on the case of $*-$ palindromic polynomial.

(1) Problem with $p=n=4$. Setting

$$
X_{2}=\left[\begin{array}{cccc}
i & 1 & 0 & 0 \\
1 & i & i & 0 \\
1 & 1 & 1 & i \\
1 & 1 & 1 & 1
\end{array}\right], \quad \Lambda_{2}=\operatorname{diag}\left(2+i, \frac{1}{2-i}, 3+2 i, 5-i\right)
$$

First, we compute the matrix $S$ by Lemma 3.1 .

$$
S=\left[\begin{array}{cccc}
4.0000+2.0000 i & 3.0000 & 4.0000 & 5.0000 \\
3.0000 & 4.0000+2.0000 i & 4.0000 & 5.0000 \\
1.2308+2.1538 i & 0.2462+0.4308 i & 3.0000+2.0000 i & 5.0000 \\
2.1154+0.5769 i & 0.4231+0.1154 i & 3.2692+1.3462 i & -10.0000+2.0000 i
\end{array}\right] .
$$


Clearly,

$$
\left\|S \Lambda_{2}^{-1}+S^{*} \Lambda_{2}-\Lambda_{2}^{-*} S^{*}-\Lambda_{2}^{*} S\right\|_{F}=6.5953 e-15,
$$

which implies that $S \in \mathbb{S}_{\Lambda_{2}}$. Hence, by $(3.9)$ we obtain that

$$
\begin{gathered}
A=\left[\begin{array}{cccc}
2.7538+3.5692 i & 3.6231+0.7154 i & 3.6692-1.9538 i & -1.9538-3.6692 i \\
1.2538+3.0692 i & 3.1231+2.2154 i & 4.1692-0.9538 i & -0.9538-4.1692 i \\
-1.0308+1.9462 i & -0.0231+3.0846 i & -5.1038+3.3808 i & 9.8808-3.3962 i \\
-4.7923-2.2615 i & -5.3154-0.1769 i & 2.3885+2.2423 i & 0.7423+6.1115 i
\end{array}\right], \\
Q=\left[\begin{array}{cccc}
-20.9538+0.0000 i & -15.7538+6.0000 i & -10.8462+15.3231 i & 28.3231+1.8462 i \\
-15.7538-6.0000 i & -15.3538+0.0000 i & -12.8462+11.7231 i & 22.7231+9.8462 i \\
-10.8462-15.3231 i & -12.8462-11.7231 i & 19.0692 & -23.0769+24.8154 i \\
28.3231-1.8462 i & 22.7231-9.8462 i & -23.0769-24.8154 i & -14.7000+0.0000 i
\end{array}\right]
\end{gathered}
$$

which satisfies

$$
\left\|Q-Q^{*}\right\|_{F}=8.1524 e-15, \quad\left\|A^{*} X_{2} \Lambda_{2}^{2}+Q X_{2} \Lambda_{2}+A X_{2}\right\|_{F}=7.4788 e-14 .
$$

(2) Problem with $p=4, n=6$. Setting

$$
X_{1}=\left[\begin{array}{cccc}
i & 1 & 0 & 0 \\
1 & i & i & 0 \\
1 & 1 & 1 & i \\
1 & 1 & 1 & 1 \\
i & i & 1 & 1 \\
1 & i & 1 & i
\end{array}\right], \quad \Lambda_{1}=\operatorname{diag}\left(2+i, \frac{1}{2-i}, 3+2 i, 5-i\right)
$$

By Theorem 3.5 we choose $A_{12}=0_{4 \times 2}, A_{21}=0_{2 \times 4}$ and $A_{22} \in \mathbb{C}^{2 \times 2}, Q_{22}^{*}=Q_{22} \in \mathbb{C}^{2 \times 2}$ randomly, and hence by Theorem 3.5 we get an $*$-palindromic polynomial $P(\lambda)=\lambda^{2} A^{*}+\lambda Q+A$ with

$$
\begin{aligned}
A= & {\left[\begin{array}{cccccc}
0.6416+0.3124 i & 0.3591-0.5271 i & -0.3242-0.1669 i & 0.2102+0.4968 i & 0.0667+0.0005 i & -0.0665-0.0895 i \\
0.2613+0.1072 i & -0.4845+0.3252 i & 1.3503-0.3880 i & -0.6792+0.8000 i & -1.0564+0.3438 i & 1.1193-0.0677 i \\
-0.2544+0.2129 i & 1.1065-0.1844 i & -0.7322+0.1091 i & 1.5206-0.6897 i & 1.1980-0.5242 i & -1.8806+0.3696 i \\
0.2475-0.4017 i & -0.7388-0.3697 i & 1.3070+0.2114 i & -0.4394+0.3511 i & -0.3709+0.4688 i & 0.6331+0.1516 i \\
0.0087+0.0462 i & -0.8670+0.2003 i & 1.0111+0.2363 i & -0.4519+0.5584 i & -0.3083+0.7723 i & 0.9515+0.4968 i \\
-0.0663-0.5214 i & 0.9636-0.1457 i & -1.6944-0.0523 i & 0.9761-0.5631 i & 1.3298-0.4966 i & -0.7525+0.5448 i
\end{array}\right], } \\
Q= & {\left[\begin{array}{cccccc}
-1.4336-0.0000 i & -0.7639-0.4813 i & -0.8220-1.5735 i & -0.8606-1.3438 i & 0.4993-0.3390 i & 2.2370+1.0839 i \\
-0.7639+0.4813 i & 3.2892-0.0000 i & -5.1537-1.1372 i & 3.3897-2.2958 i & 3.7315-1.8977 i & -4.4318+0.4333 i \\
-0.8220+1.5735 i & -5.1537+1.1372 i & 7.3190 & -5.3802+1.8705 i & -4.2254+1.7259 i & 6.4811-0.0277 i \\
-0.8606+1.3438 i & 3.3897+2.2958 i & -5.3802-1.8705 i & 1.0328-0.0000 i & 2.4472-1.0791 i & -4.1064-4.1146 i \\
0.4993+0.3390 i & 3.7315+1.8977 i & -4.2254-1.7259 i & 2.4472+1.0791 i & -0.3073+0.0000 i & -5.5628-3.7614 i \\
2.2370-1.0839 i & -4.4318-0.4333 i & 6.4811+0.0277 i & -4.1064+4.1146 i & -5.5628+3.7614 i & 5.1542
\end{array}\right] }
\end{aligned}
$$

which satisfy

$$
\left\|Q-Q^{*}\right\|_{F}=5.4475 e-15, \quad\left\|A^{*} X \Lambda^{2}+Q X \Lambda+A X\right\|_{F}=8.1220 e-14 .
$$

Example 4.3. This example illustrates the performance of Theorem 3.7 for solving Problem PIEP with $n=3$ and $p=5$. We only concentrate on the case of $T$-palindromic polynomial. 
Let

$$
X=\left[\begin{array}{ccccc}
1 & 0 & 0 & 1 & -1 \\
0 & 1 & 0 & 1 & 0 \\
0 & 0 & 1 & 1 & 1
\end{array}\right], \quad \Lambda=\operatorname{diag}\left\{i, \frac{1}{i}, 1+i, \frac{1}{1+i}, 2 i\right\}
$$

Clearly, this is the case in which $n=3$ and $p=p_{\max }=5$. We can see from (3.33) that the solution has one degree of freedom. Let $X=\left[X_{1}, X_{2}\right]$ and $\Lambda^{\prime}=\operatorname{diag}\{i, 1 / i, 1+i\}$, where $X_{1} \in \mathbb{C}^{3 \times 3}$. Obviously, $X_{1}$ is the $3 \times 3$ identity matrix. We find the solution $S_{11}$ of (3.27) and 3.28 over $\mathbb{S}_{\Lambda^{\prime}}$ as follows:

$$
S_{11}=\left[\begin{array}{ccc}
(i+1) u & \frac{3-5 i}{2} u & (1-i) u \\
(5 i-3) u & \frac{21-i}{2} u & 0 \\
u & 0 & -u i
\end{array}\right]
$$

where $u \in \mathbb{C}$ is arbitrary. We can see from (3.24) and (3.32) that the solutions to the problem can be represented as $A(u)=X_{1}^{-\top} S_{11} X_{1}^{-1}=S_{11}$ and

$$
Q(u)=-X_{1}^{\top}\left(S_{11}^{\top} \Lambda^{\prime}+S_{11} \Lambda^{\prime-1}\right) X_{1}^{-1}=\left[\begin{array}{ccc}
0 & -\frac{15+9 i}{2} u & -u \\
-\frac{15+9 i}{2} u & 0 & 0 \\
-u & 0 & \frac{3 i-1}{2} u
\end{array}\right]
$$

which satisfy

$$
A(u)^{\top} X \Lambda^{2}+Q(u) X \Lambda+A(u) X=0 \quad \text { for any } u \in \mathbb{C} .
$$

It is easy to see that $\operatorname{det}(A(u))=(-15+8 i) u$, which implies that $A(u)$ is nonsingular for any nonzero $u \in \mathbb{C}$. And in this case the resulting polynomial $P(\lambda)$ is regular.

Example 4.4. In this example, we consider the inverse eigenvalue problem for the PQEP of fast train with the matrices $A$ and $Q$ given by

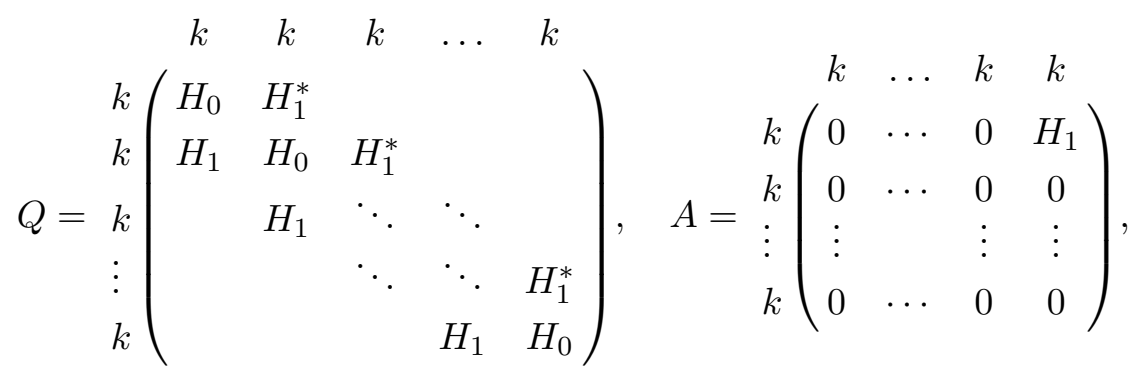

where $Q$ and $A$ both have $m$ block-rows and columns, $n=m k[7,22,23]$. The finite element method generates real $k \times k$ matrices $K_{i}$ and $M_{i}$ to give

$$
H_{i}=K_{i}+\iota \omega D_{i}-\omega^{2} M_{i} \text { with } D_{i}=c_{1} M_{i}+c_{2} K_{i}
$$


for $i=0,1$, where $\iota$ is the imaginary unit, $\omega>0$ is the frequency of the external excitation force, $c_{1}$ and $c_{2}$ are two positive parameters.

As in $7,22,23]$, we take

$$
(k, m)=(159,11),(303,19) .
$$

We choose $p(p \ll n)$ finite and nonzero eigenvalue pairs $\left(\lambda, 1 / \lambda^{*}\right)$ of $P(\lambda)=\lambda^{2} A^{*}+\lambda Q+A$ and their corresponding eigenvectors. By Theorem 2.4, we assume that $V$ is an $n \times n$ identity matrix and choose $Y_{2} \in \mathbb{C}^{n \times(n-p)}$ randomly, and get the regular $*$-palindromic polynomial $\widehat{P}(\lambda)=\lambda^{2} \widehat{A}^{*}+\lambda \widehat{Q}+\widehat{A}$. The numerical results are given by Table 4.1, where Res. stands for

$$
\text { Res. }=\left\|\widehat{A}^{*} X \Lambda^{2}+\widehat{Q} X \Lambda+\widehat{A} X\right\|_{F}
$$

\begin{tabular}{|c|c|c|c|c|c|}
\hline \multicolumn{6}{|c|}{$\omega=1180, c_{1}=0.2, c_{2}=0.8$} \\
\hline$(k, m)$ & & $p=8$ & $p=12$ & $p=20$ & $p=40$ \\
\hline$(159,11)$ & Res. & $6.5354 e-14$ & $7.9433 e-14$ & $9.2483 e-14$ & $1.3454 e-13$ \\
$(303,19)$ & & $1.3660 e-13$ & $1.6870 e-13$ & $1.6581 e-13$ & $2.3886 e-13$ \\
\hline \multicolumn{7}{|c|}{$\omega=5000, c_{1}=0.3, c_{2}=0.7$} \\
\hline$(159,11)$ & Res. & $8.1584 e-16$ & $1.1376 e-15$ & $1.4937 e-15$ & $2.1897 e-15$ \\
$(303,19)$ & & $1.2413 e-15$ & $1.3089 e-15$ & $1.8344 e-15$ & $2.3786 e-15$ \\
\hline
\end{tabular}

Table 4.1: Numerical results of Example 4.4

We can see from Table 4.1 that the prescribed eigenvalues and eigenvectors are reproduced successfully by the new polynomial $\widehat{P}(\lambda)$. And we also should point out that the coefficient matrices $\widehat{A}$ and $\widehat{Q}$ obtained by our method do not have the sparsity structure 4.1). How to obtain the structural solution to the Problem PIEP of the fast train is our further investigation.

\section{Conclusion}

In this paper, we consider the inverse eigenvalue problems of the quadratic palindromic systems with $p(1 \leq p \leq 2 n)$ prescribed eigenvalues and eigenvectors. We proposed two different methods to solve the inverse problem for different $p$. We also give some sufficient conditions under which the inverse problem has the regular solution. Generally, the matrices of palindromic system often have special structure 22, 23], for example, 
symmetry and sparsity and so on. How to get the structural solution to the inverse eigenvalue problems of the palindromic system is the subject of further investigation.

\section{Acknowledgments}

The authors would like to express their great thankfulness to the referees for the comments and constructive suggestions very much, which are valuable in improving the quality of the original paper. The research is financially supported by Hunan Provincial Key Laboratory of Mathematical Modeling and Analysis in Engineering (Changsha University of Science \& Technology).

\section{References}

[1] M. Al-Ammari, Analysis of Structured Polynomial Eigenvalues Problems, Phd thesis, The University of Manchester, Manchester, UK, 2011.

[2] Z. Bai, M. Chen and X. Yuan, Applications of the alternating direction method of multipliers to the semidefinite inverse quadratic eigenvalue problem with a partial eigenstructure, Inverse Problems 29 (2013), no. 7, 075011, 27 pp.

[3] Z.-J. Bai, D. Chu and D. Sun, A dual optimization approach to inverse quadratic eigenvalue problems with partial eigenstructure, SIAM J. Sci. Comput. 29 (2007), no. $6,2531-2561$.

[4] L. Batzke and C. Mehl, On the inverse eigenvalue problem for T-alternating and T-palindromic matrix polynomials, Linear Algebra Appl. 452 (2014), 172-191.

[5] Y. Cai and J. Qian, On some inverse eigenvalue problems of quadratic palindromic systems, arXir:1606.03840v1.

[6] D. Chu, M. Chu and W.-W. Lin, Quadratic model updating with symmetric, positive definiteness, and no spill-over, SIAM J. Matrix Anal. Appl. 31 (2009), no. 2, 546-564.

[7] E. K.-W. Chu, T.-M. Hwang, W.-W. Lin and C.-T. Wu, Vibration of fast trains, palindromic eigenvalue problems and structure-preserving doubling algorithms, J. Comput. Appl. Math. 219 (2008), no. 1, 237-252.

[8] _ Palindromic eigenvalue problems: a brief survey, Taiwanese J. Math. 14 (2010), no. 3A, 743-779.

[9] M. T. Chu, B. Datta, W.-W. Lin and S. Xu, Spillover phenomenon in quadratic model updating, AIAA J. 46 (2008), no. 2, 420-428. 
[10] M. T. Chu, Y.-C. Kuo and W.-W. Lin, On inverse quadratic eigenvalue problems with partially prescribed eigenstructure, SIAM J. Matrix Anal. Appl. 25 (2004), no. 4, 9951020.

[11] M. T. Chu, W.-W. Lin and S.-F. Xu, Updating quadratic models with no spillover effect on unmeasured spectral data, Inverse Problems 23 (2007), no. 1, 243-256.

[12] B. N. Datta and V. Sokolov, A solution of the affine quadratic inverse eigenvalue problem, Linear Algebra Appl. 434 (2011), no. 7, 1745-1760.

[13] G. H. Golub and C. F. Van Loan, Matrix Computations, Third edition, Johns Hopkins Studies in the Mathematical Science, Johns Hopkins University Press, Baltimore, MD, 1996.

[14] C. H. Guo and W.-W. Lin, Solving a structured quadratic eigenvalue problem by a structure-preserving doubling algorithm, SIAM J. Matrix Anal. Appl. 31 (2010), no. 5, $2784-2801$.

[15] N. J. Higham, F. Tisseur and P. M. Van Dooren, Detecting a definite Hermitian pair and a hyperbolic or elliptic quadratic eigenvalue problem, and associated nearness problems, Linear Algebra Appl. 351 (2002), 455-474.

[16] A. Hilliges, Numerische Lösung von quadratischen Eigenwertproblemen mit Anwendung in der Schienendynamik, Diplomarbeit, Technical University Berlin, Inst. Für Mathematik, Germany, 2004.

[17] A. Hilliges, C. Mehl and V. Mehrmann, On the solution of palindromic eigenvalue problems, in: 4th European Congress on Computational Methods in Applied Sciences and Enginnerings (ECCOMAS), Jyväskylä, Finland, 2004.

[18] I. C. F. Ipsen, Accurate Eigenvalues for Fast Trains, SIAM News 37, SIAM, Philadelphia, 2004.

[19] D. Kressner, C. Schröder and D. S. Watkins, Implicit QR algorithms for palindromic and even eigenvalue problems, Numer. Algorithms 51 (2009), no. 2, 209-238.

[20] P. Lancaster, Model-updating for self-adjoint quadratic eigenvalue problems, Linear Algebra Appl. 428 (2008), no. 11-12, 2778-2790.

[21] R.-C. Li, W.-W. Lin and C.-S. Wang, Structured backward error for palindromic polynomial eigenvalue problems, Numer. Math. 116 (2010), no. 1, 95-122. 
[22] L. Lu, T. Wang, Y.-C. Kuo, R.-C. Li and W.-W. Lin, A fast algorithm for fast train palindromic quadratic eigenvalue problems, SIAM J. Sci. Comput. 38 (2016), no. 6, A3410-A3429.

[23] L. Lu, F. Yuan and R.-C. Li, A new look at the doubling algorithm for a structured palindromic quadratic eigenvalue problem, Numer. Linear Algebra Appl. 22 (2015), no. 3, 393-409.

[24] D. S. Mackey, N. Mackey, C. Mehl and V. Mehrmann, Vector spaces of linearizations for matrix polynomials, SIAM J. Matrix Anal. Appl. 28 (2006), no. 4, 971-1004.

[25] _ Structured polynomial eigenvalue problems: Good vibrations from good linearizations, SIAM J. Matrix Anal. Appl. 28 (2006), no. 4, 1029-1051.

[26] _ Numerical methods for palindromic eigenvalue problems: computing the antitriangular Schur form, Numer. Linear Algebra Appl. 16 (2009), no. 1, 63-86.

[27] C. Schröder, Urv decomposition based structured methods for palindromic and even eigenvalue problems, TU Berlin Germany, Preprint 375 (2007).

[28] _ Palindromic and Even Eigenvalue Problems-analysis and Numerical Methods, Ph.D dissertation, Technical University Berlin, Germany, 2008.

[29] S. Zaglmayr, Eigenvalue Problems in SAW-Filter Simulations, Diplomarbeit, Institute of Computational Mathematics, Johannes Kepler University Linz, Austria, 2002.

[30] K. Zhao, L. Cheng, S. Li and A. Liao, A new updating method for the damped massspring systems, Appl. Math. Model. 62 (2018), 119-113.

[31] K. Zhao, L. Cheng and A. Liao, Updating $\star$-palindromic quadratic systems with no spill-over, Comput. Appl. Math. 37 (2018), no. 5, 5587-5608.

[32] K. Zhao and G. Yao, Application of the alternating direction method for an inverse monic quadratic eigenvalue problem, Appl. Math. Comput. 244 (2014), 32-41.

Kang Zhao

School of Mathematics and Statistics, Changsha University of Science and Technology,

Changsha 410114, P. R. China

and

College of Science, National University of Defense Technology, Changsha 410073,

P. R. China

E-mail address: zhaokangmath@126.com 


\section{Lizhi Cheng}

College of Science, National University of Defense Technology, Changsha 410073, P. R. China

E-mail address: clzcheng@nudt.edu.cn

\section{Anping Liao}

College of Mathematics and Econometrics, Hunan University, Changsha 410082,

P. R. China

E-mail address: liaoap@hnu.cn

Shengguo Li

College of Computer, National University of Defense Technology, Changsha 410073, P. R. China

E-mail address: shengguolsg@126.com 